\title{
Riqueza y diversidad de especies leñosas del bosque tropical caducifolio EI Tarimo, Cuenca del Balsas, Guerrero
}

\author{
Richness and diversity of woody species in the tropical dry forest of El Tarimo, Cuenca del \\ Balsas, Guerrero
}

\author{
Fernando Pineda-García ${ }^{1}$, Libertad Arredondo-Amezcua ${ }^{1}$ y Guillermo Ibarra-Manríquez ${ }^{1 *}$ \\ ${ }^{1}$ Centro de Investigaciones en Ecosistemas, Universidad Nacional Autónoma de México. Antigua carretera a Pátzcuaro 8701. Col. Ex-Hacienda de \\ San José de La Huerta,. 58190. Morelia, Michoacán, México. \\ "Correspondencia: gibarra@oikos.unam.mx, giman@servidor.unam.mx
}

\begin{abstract}
Resumen. Se describe la composición florística, la riqueza y la diversidad de especies de un bosque tropical caducifolio en la provincia florística Cuenca del Balsas, México. Se seleccionaron 4 sitios de $1000 \mathrm{~m}^{2}$ cada uno, censándose los árboles, arbustos y lianas con d.a.p. $\geq 1 \mathrm{~cm}$. En total se registraron 1456 individuos, pertenecientes a 82 especies, 56 géneros y 24 familias. Independientemente del sitio y de la forma de crecimiento, Leguminosae fue la familia con mayor número de especies y de individuos. Los géneros más diversos fueron Bursera (Burseraceae) y Cordia (Boraginaceae) con 9 y 4 especies, respectivamente. La riqueza entre los sitios varió de 43 a 55 especies y su similitud fue más alta en el nivel de familia que en el de especie. Los árboles fueron la forma de crecimiento con mayor riqueza de especies. Respecto a otros bosques tropicales caducifolios de México y del mundo, los sitios que se estudiaron en este bosque ocupan una posición baja en cuanto a sus valores de riqueza y estructura.
\end{abstract}

Palabras clave: árboles, composición florística, diversidad, lianas.

\begin{abstract}
Floristic composition, species richness, and diversity of the seasonally dry tropical forest in the floristic province of the Balsas Depression, México, is described. We sampled four 1,000 $\mathrm{m}^{2}$ sites and recorded species and dbh of trees, shrubs and lianas $\geq 1 \mathrm{~cm}$ dbh. Data from 1,456 individuals were recorded, representing 82 species, 56 genera, and 24 families. Independently of site or growth form, Leguminosae was the family with the highest number of species and individuals. Bursera (Burseraceae) and Cordia (Boraginaceae) were the most speciose genera, with nine and four species, respectively. Species richness among sites ranged from 43-55 species and their similarity was higher at the family level than the species level. Trees had higher numbers of species than shrubs and lianas. Our results indicate that these forests have low values of species richness and structure attributes in relation with other tropical dry forests of Mexico and the world.
\end{abstract}

Key words: Diversity, floristic composition, lianas, trees.

\section{Introducción}

En México, el bosque tropical caducifolio (BTC) o selva baja caducifolia (Gentry, 1942; Miranda y Hernández-X, 1963; Rzedowski, 1978; Challenger, 1998; Pennigton y Sarukhán, 1998), se distribuia principalmente a lo largo de la vertiente del Pacífico, y de manera discontinua hacia la región central del país y por la vertiente del Golfo de México; prácticamente cubría el $17 \%$ de su territorio

Recibido: 14 diciembre 2005; aceptado: 21 noviembre 2006
(Rzedowski, 1978). Trejo y Dirzo (2000) estiman que sólo $72900 \mathrm{~km}^{2}(3.7 \%)$ permanece sin alteración notoria.

La tasa de deforestación anual para el BTC es alta, entre $1.4 \%$ y 2\% (Masera et al., 1997; Trejo y Dirzo, 2000). Lo anterior es preocupante si se considera que es un tipo de vegetación con un notable legado biótico; por ejemplo, se ha calculado que el BTC alberga al menos el 33\% (824 especies) de los vertebrados terrestres del territorio mexicano y cerca de 6000 especies de plantas vasculares (Ceballos y García, 1995; Rzedowski, 1991a). $\mathrm{Si}$ se consideran los bosques tropicales caducifolios en conjunto con los tropicales subcaducifolios y espinosos, 
aproximadamente el $40 \%$ de sus especies de plantas vasculares se localizan exclusivamente en la República Mexicana (Rzedowski, 1991b). Desde el punto de vista faunístico, Ceballos y García (1995) mencionan que 90 especies de vertebrados endémicos de México restringen su distribución a este tipo de vegetación.

Los estudios para caracterizar la composición, estructura y riqueza de especies del BTC en diversas localidades del planeta revelan una gran heterogeneidad (Murphy y Lugo, 1986; Ray et al., 1998; Martínez-Yrízar et al., 2000; Balvanera et al., 2002; Phillips y Miller, 2002; White y Hood, 2004; Lot y Atkinson, 2006). Con base en censos realizados para árboles, arbustos y lianas con un diámetro $\geq 2.5$ en parcelas de 0.1 ha (Gentry, 1995; Trejo, 1998; Phillips y Miller, 2002; Gillespie et al., 2000; Trejo y Dirzo, 2002; Gillespie y Jaffré, 2003), es posible destacar lo siguiente: i) 2 localidades de Bolivia (Río Negro y Nuevo Mundo) revelan los valores más altos de riqueza, con 169 y 149 especies, respectivamente, ii) el área basal para diferentes localidades oscila entre 1.0 y $10.3 \mathrm{~m}^{2}$, esta última registrada en Las Flores, Tamaulipas, México, y iii) con muy raras excepciones, Bignoniaceae, Leguminosae, Euphorbiaceae Rubiaceae, y Sapindaceae se distinguen por ser las familias con más especies.

La provincia florística Cuenca del Balsas (Rzedowski, 1978) es un área que destaca por la riqueza y grado de endemismo de su flora (Rzedowski, 1991a, b; Challenger, 1998). Esta región se encuentra predominantemente cubierta por BTC. La mayoría de los estudios de vegetación realizados en esta zona son de tipo descriptivo (Miranda, 1941, 1947; Miranda y Hernández-X., 1963; Rzedowski, 1978; Pennigton y Sarukhán, 1998). Recientemente, Trejo (1998) aporta información sobre la estructura de 4 sitios de 0.1 ha de BTC localizados en esta región, para lo cual censaron los árboles y lianas $\geq 1 \mathrm{~cm}$ d.a.p. Estos sitios difieren en los atributos florísticos y estructurales de las comunidades que albergan; por ejemplo, respecto al número de taxa (26-38 familias, 54-86 géneros y 76-115 especies), número de individuos (443-748) o área basal (3.47-7.01 $\left.\mathrm{m}^{2}\right)$. Si se toma en cuenta el tamaño de esta provincia florística $\left(\approx 112320 \mathrm{~km}^{2}\right)$ y la variación que presenta en general el BTC en sus atributos de composición florística, es recomendable incrementar en la región el número de sitios donde se obtenga información florística, de riqueza y diversidad de especies vegetales, para caracterizarla de manera más adecuada.

En consecuencia, el presente estudio describe la riqueza y diversidad de la flora leñosa (árboles, arbustos y lianas) del BTC de El Tarimo, una localidad ubicada en la provincia florística Cuenca del Balsas en Guerrero, para determinar su grado de similitud respecto a otras localidades de México y del resto del mundo.

\section{Materiales y métodos}

Área de estudio. El Tarimo, comoes conocida regionalmente la zona de estudio, se localiza en el municipio de Zirándaro, Guerrero, México, en la subprovincia de la depresión del Balsas (Fig. 1), de la provincia morfotectónica de la Sierra Madre del Sur, donde dominan cuerpos sedimentarios del Mesozoico (Ferrusquía-Villafranca, 1998; INEGI, 2001). Los litosoles son el tipo de suelo predominante en el área, característicos por su escasa profundidad, desarrollo incipiente, baja permeabilidad y moderada fertilidad (SPP, 1983; INEGI, 1989). Tomando en cuenta los datos climáticos de la estación meteorológica más cercana al sitio de estudio (Aratichanguío), el clima en el área es cálido subhúmedo, con régimen de lluvias de verano $\mathrm{Aw}_{\mathrm{o}}(\mathrm{w})(\mathrm{e}) \mathrm{g}$; la temperatura media anual es de $29.4^{\circ} \mathrm{C}$, registrándose los meses más cálidos de marzo a mayo y una precipitación promedio anual de $872.3 \mathrm{~mm}$, que se
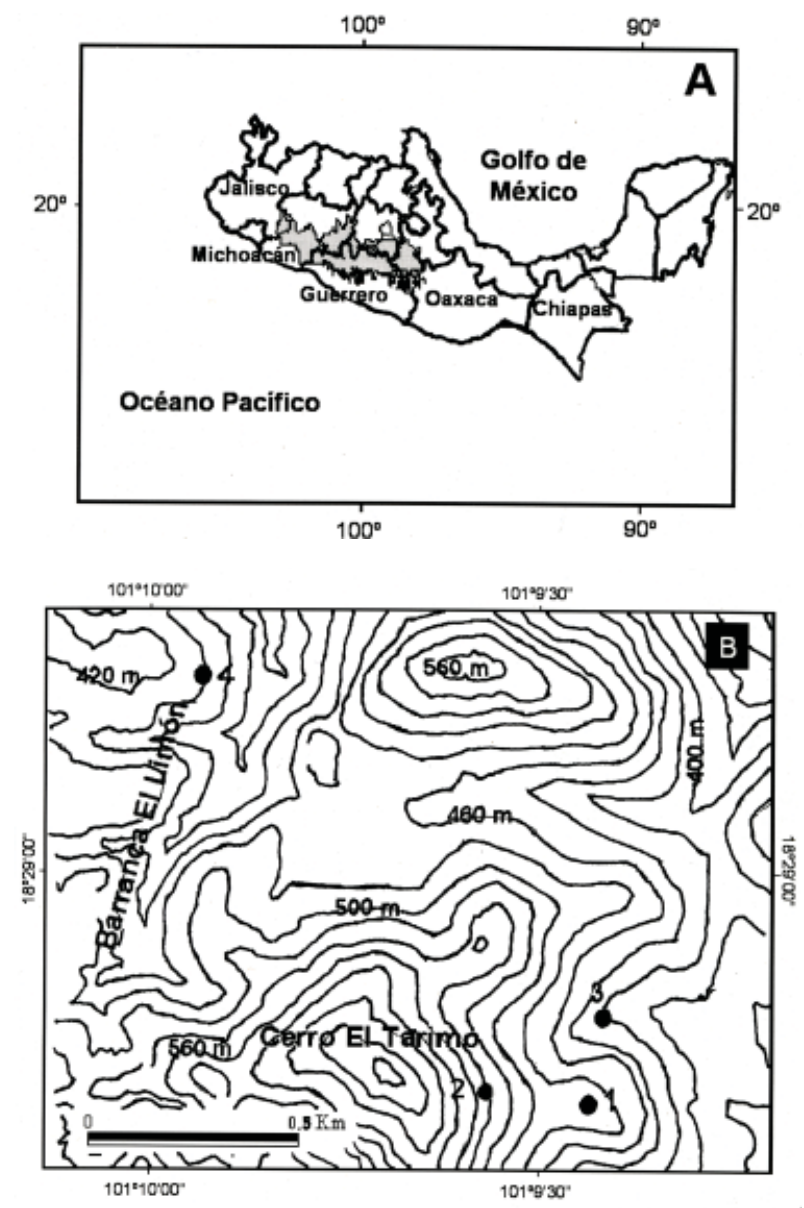

Figura 1. A) Localización de la provincia florística Cuenca del Balsas, Guerrero (área sombreada), México (Rzedowski, 1978), B ) Localización de los sitios de muestreo (•). 
distribuye de junio a octubre (García, 1981). Miranda (1947) describe de manera general la vegetación del BTC en la Cuenca del Balsas, donde reconoce 3 principales variantes: i) cuajiotales, en la que predominan especies del género Bursera (p. ej. B. copallifera (DC.) Bullock, B. fagaroides (Kunth) Engl. o B. grandifolia (Schltdl.) Engl.), junto con Amphipterygium adstringens Schiede ex Schltdl., Ceiba parvifolia Rose, Comocladia engleriana Loes., Cyrtocarpa procera Kunth y Lysiloma microphyllum Benth., ii) tecomaxochitlales, donde se combinan especies del género Bursera con Cochlospermum vitifolium (Willd.) Spreng., Pseudobombax ellipticum (Kunth) Dugand, Sideroxylon capiri (A. DC.) Pittier y Swietenia humilis Zucc., y iii) tetlales, ubicados en localidades más secas y teniendo como especie dominante a Pseudosmodingium perniciosum (Kunth) Engl.

Para caracterizar la vegetación del BTC en El Tarimo se seleccionaron 4 sitios lo mejor conservados posible (Fig. 1a). Para cumplir con este criterio, se evitaron localidades con un disturbio antropogénico aparente, lo cual fue detectado por la presencia de rastros de ganado (excremento o huellas) o troncos cortados. El método de muestreo que se utilizó fue el propuesto por Gentry (1982) para censos de especies leñosas con $\geq 2.5 \mathrm{~cm}$ de diámetro a la altura del pecho (d.a.p.) presentes en los bosques tropicales del Neotrópico (Gentry, 1982, 1995; Phillips y Miller, 2002). Este método consiste en colocar 10 líneas de $50 \times 2 \mathrm{~m}$ ( $0.1 \mathrm{ha})$; en el presente trabajo, se alinearon de manera paralela, con una separación aproximada de 20 m entre sí. Se trabajó con la modificación del método de Gentry (1982) propuesta por Trejo (1998), donde se miden e identifican las plantas leñosas (árboles y arbustos) enraizadas dentro de cada línea, con un d.a.p. de $\geq 1 \mathrm{~cm}$ (aproximadamente a $1.4 \mathrm{~m}$ del suelo); también se incluyeron las especies de lianas, cuyo diámetro se midió en la base del tronco. Las especies con un solo tronco a nivel del suelo se clasificaron como árboles; aquellas con ramificaciones desde su base, con dos o más ejes de crecimiento leñoso, como arbustos, y las plantas leñosas que, a diferencia de las anteriores formas de crecimiento, son mecánicamente dependientes, como lianas. Las especies se determinaron en el campo y en caso de incertidumbre sobre su identificación se colectaron ejemplares para su revisión posterior. Los ejemplares colectados fueron identificados en el herbario IEB del Instituto de Ecología en Pátzcuaro, Michoacán y para ello se consultaron floras o monografías taxonómicas.

El análisis de la diversidad de cada sitio se estimó, usando primeramente el número de especies (riqueza de especies $S$ ). De acuerdo con las sugerencias de Magurran (2003), la diversidad de los sitios se evaluó calculando el índice alfa $(\alpha)$ de la serie logarítmica:

$$
\alpha=\mathrm{N}(1 \mathrm{x}) / \mathrm{x}
$$

donde $\mathrm{N}$ es el número total de individuos registrados en el sitio de muestreo y x se obtiene a partir de la siguiente fórmula:

$$
\mathrm{S} / \mathrm{N}=[(1-\mathrm{x}) / \mathrm{x}][-\ln (1-\mathrm{x})]
$$

La similitud a nivel de especies y familias entre las cuatro parcelas se calculó con el índice de Jaccard (CJ), utilizando la presencia-ausencia de los taxa:

$$
\mathrm{CJ}=\mathrm{a} / \mathrm{a}+\mathrm{b}+\mathrm{c}
$$

donde a es el número de especies compartidas entre 2 sitios, b es el número de especies presentes sólo en el sitio 1 y c el número de especies localizadas exclusivamente en el sitio 2 .

\section{Resultados}

En El Tarimo, 43 (52.4\%) especies, más de la mitad, fueron árboles, mientras que los arbustos y lianas presentaron una riqueza similar, $22(26.8 \%)$ y 17 (20\%) especies, respectivamente (Apéndice 1). De manera

Cuadro 1. Riqueza total de especies y por forma de crecimiento

\begin{tabular}{|c|c|c|c|}
\hline Sitio & $\begin{array}{c}\text { Árboles y } \\
\text { arbustos }\end{array}$ & Lianas & Total \\
\hline \multicolumn{4}{|c|}{ Categoría diamétrica $\geq 1 \mathrm{~cm}$} \\
\hline 1 & 34 & 9 & 43 \\
\hline 2 & 35 & 9 & 44 \\
\hline 3 & 42 & 13 & 55 \\
\hline 4 & 36 & 11 & 47 \\
\hline Total & 66 & 18 & 82 \\
\hline \multicolumn{4}{|c|}{ Categoría diamétrica $\geq 2.5 \mathrm{~cm}$} \\
\hline 1 & 29 & 7 & 36 \\
\hline 2 & 31 & 9 & 40 \\
\hline 3 & 39 & 10 & 50 \\
\hline 4 & 30 & 10 & 40 \\
\hline Total & 55 & 16 & 72 \\
\hline \multicolumn{4}{|c|}{ Categoría diamétrica $\geq 10 \mathrm{~cm}$} \\
\hline 1 & 18 & 4 & 22 \\
\hline 2 & 19 & 1 & 20 \\
\hline 3 & 17 & 3 & 20 \\
\hline 4 & 15 & 0 & 15 \\
\hline Total & 34 & 6 & 40 \\
\hline
\end{tabular}
en los sitios de estudio con base en diferentes categorías diamétricas.

Los números con negritas indican los valores más altos para cada categoría. 
consistente entre los sitios y entre las diferentes categorías diamétricas, las lianas muestran siempre un menor número de especies que los árboles y arbustos (Cuadro 1). En los 4 sitios muestreados se registraron 82 especies; 67 (81.7\%) fueron identificadas hasta el nivel de especie, mientras que a $8(9.8 \%)$ y $5(6 \%)$ se les asignó categoría genérica y de familia, respectivamente, y sólo se registraron 2 morfoespecies (Apéndice 1).

El sitio 3 fue el que presentó mayor número de especies en las categorías diamétricas $\geq 1 \mathrm{~cm}$ de d.a.p. (55 especies) $\mathrm{y} \geq 2.5 \mathrm{~cm}$ (50), en tanto que el sitio 1 , con 22 especies, lo fue para la categoría diamétrica $\geq 10 \mathrm{~cm}$ (Cuadro 1). El índice $\alpha$ para cada uno de los sitios (1-4) mostró los siguientes valores: $13.1,12.4,16.9$, y 16 respectivamente. En el nivel de especie, de acuerdo al índice de Jaccard, los sitios 1 y 3 son los más similares, con valores de similitud de 0.58 y los sitios menos parecidos fueron el 2 y el 4 con 0.4 ( Cuadro 2). De las 82 especies registradas en este estudio, $21(25.6 \%)$ estuvieron representadas en todos los sitios y $30(36.6 \%)$ únicamente en un sitio (Apéndice 1).

El número total de familias censadas en el trabajo fue de 24 (Apéndice 1); el valor mínimo (17) se presentó en el sitio 1, y el máximo en el 3 (22). El mayor número de especies fue de Leguminosae (25), seguida por Burseraceae (9), Rubiaceae (6), Anacardiaceae (4) y Boraginaceae (4), las cuales concentran el $58.5 \%$ de las especies; 13 familias estuvieron representadas por 2 especies y 6 por una sola (Apéndice 1). Leguminosae también mostró la mayor riqueza de especies para las 3 formas de crecimiento incluidas en el estudio (11, 9 y 5 especies para árboles, arbustos y lianas, respectivamente). En el caso de los árboles, a Leguminosae la siguen en orden de importancia Burseraceae ( 9 especies), Boraginaceae y Anacardiaceae (ambas con 4 especies). Respecto a los arbustos, el segundo lugar lo ocupa Rubiaceae (4), seguida de Erythroxylaceae (2). En cuanto a las lianas, 5 familias, Asclepiadaceae, Convolvulaceae, Compositae, Sapindaceae y Vitaceae,

Cuadro 2. Valores del índice de Jaccard a nivel de familias (diagonal superior) y especies $\geq 1 \mathrm{~cm}$ d.a.p (diagonal inferior) en los sitios incluidos en el presente trabajo. Valores más altos en negritas.

\begin{tabular}{ccccc}
\hline Sitio & 1 & 2 & 3 & 4 \\
\hline 1 & - & 0.75 & 0.69 & 0.6 \\
2 & 0.50 & - & 0.66 & 0.81 \\
3 & $\mathbf{0 . 5 8}$ & 0.50 & - & $\mathbf{0 . 8 2}$ \\
4 & 0.47 & 0.40 & 0.41 & - \\
\hline
\end{tabular}

comparten la segunda posición, con 2 especies cada una. Los sitios más parecidos respecto a su composición de familias son el 3 y el 4 , con un índice de similitud de Jaccard de 0.82 , seguido inmediatamente por los sitios 2 y 4, con 0.81 (Cuadro 2). Un poco más de la mitad de familias $(14,58.3 \%)$ fueron censadas en todas las parcelas, en tanto que Moraceae se restrigió únicamente al sitio 3.

El sitio 4 mostró un número muy bajo de individuos (285) en comparación con los otros sitios censados, sobre todo para el 2 y 3, con 417 y 419 individuos (Cuadro 3). En los sitios 1, 3 y 4 Randia capitata fue la especie que tuvo el mayor número de individuos (61, 33 y 48, respectivamente) y en el sitio 2 , Serjania triquetra con 58 individuos (Cuadro 3). En este último sitio, la segunda posición por su abundancia fue también para una especie de liana (Otopappus mexicanus); para este atributo, Erytroxylum rotundifolium es la única especie que se ubica entre las primeras 5 posiciones en los 4 sitios.

Finalmente, el área basal cuantificada entre los 4 sitios fue similar, con un intervalo de 2.67 a $3.47 \mathrm{~m}^{2}$ ( Cuadro 3). En los sitios 1 y 4 Cyrtocarpa procera presentó la mayor área basal con 1.57 y $1.14 \mathrm{~m}^{2}$, respectivamente; en el sitio 2, Bursera fagaroides y en el sitio 3, Cochlospermum vitifolium (Cuadro 3). La única especie registrada entre las 5 más importantes por su área basal para los 4 sitios fue Cordia eleagnoides, mientras que en el nivel de género únicamente Bursera presenta este patrón de distribución local. Con excepción del sitio 4, no se registran especies de Leguminosae entre las 5 más importantes por el número de individuos o por el área basal.

\section{Discusión}

El género con el mayor número de especies en El Tarimo fue Bursera (9 especies), uno de los géneros arbóreos con mayor número de especies del BTC en México (Cuevas et al., 1998; Trejo, 1998; Zepeda y Velázquez, 1999; Salas-Morales, 2002; Lott y Atkinson, 2006). El BTC de la Cuenca del Balsas está considerado como un centro de diversificación y endemismo de este género (Miranda, 1947; Rzedowski, 1978, 1991a, b; Rzedowski et al., 2005). Gentry (1995) indica que la mayor proporción de especies de Bursera y Caesalpinia en el BTC de México (éste último género también está presente en el área estudiada), permite definirla como una región biogeográfica de todo el Neotrópico. Entre los géneros con más amplia distribución en el BTC de América, en El Tarimo se encuentran Acacia, Bauhinia, Bursera, Cordia, Erythroxylum, Ficus, Hippocratea, Pithecellobium, Randia, Serjania y Tabebuia, destacándose la ausencia de Capparis, Casearia, Croton, Eugenia, Macfadyena, Trichilia y Zanthoxylum, lo cual, 
Cuadro 3. Especies con mayor número de individuos ( $\geq 1 \mathrm{~cm}$ d.a.p.) y área basal $\left(\mathrm{m}^{2}\right)$ en los cuatro sitios muestreados en la zona de estudio.

\begin{tabular}{lclc}
\hline Especie & Individuos (\%) & Especie & Área Basal (\%) \\
\hline Sitio 1 & & & \\
Randia capitata & $61(18.2)$ & Cyrtocarpa procera & $1.57(45.5)$ \\
Cyrtocarpa procera & $31(9.2)$ & Bursera fagaroides & $0.39(11.4)$ \\
Cordia eleagnoides & $29(8.6)$ & Cordia eleagnoides & $0.31(8.9)$ \\
Erythroxylum rotundifolium & $23(6.9)$ & Cochlospermum vitifolium & $0.21(6.3)$ \\
Serjania triquetra & $19(5.7)$ & Amphypterigium adstringens & $0.12(3.6)$ \\
Total & $335(100)$ & Total & $3.47(100)$ \\
Sitio 2 & & & \\
Serjania triquetra & $58(13.8)$ & Bursera fagaroides & \\
Otopappus mexicanus & $34(8.1)$ & Cordia eleagnoides & $0.33(12.2)$ \\
Bursera fagaroides & $33(8)$ & Amphypterigium adstringens & $0.31(11.6)$ \\
Erythroxylum rotundifolium & $29(7)$ & Vitex pyramidata & $0.25(9.4)$ \\
Cordia eleagnoides & $25(6)$ & Erythroxylum rotundifolium & $0.23(8.8)$ \\
Total & $419(100)$ & Total & $0.18(6.7)$ \\
Sitio 3 & & & $2.73(100)$ \\
Randia capitata & $33(8)$ & Cochlospermum vitifolium & \\
Erythroxylum rotundifolium & $32(7.7)$ & Cordia eleagnoides & $0.89(28.3)$ \\
Otopappus mexicanus & $26(6.2)$ & Bursera velatina & $0.24(7.5)$ \\
Hippocratea celastroides & $24(6)$ & Bursera grandiflora & $0.19(6)$ \\
Lysiloma tergeminum & $23(5.5)$ & Pseudobombax ellipticum & $0.17(5.6)$ \\
Total & $417(100)$ & Total & $0.15(5)$ \\
Sitio 4 & & & $3.18(100)$ \\
Randia capitata & $48(16.8)$ & Cyrtocarpa procera & \\
Phitecellobium lanceolatum & $33(11.6)$ & Cordia eleagnoides & \\
Cordia eleagnoides & $26(9.1)$ & Phitecellobium lanceolatum & $0.14(43)$ \\
Erythroxylum rotundifolium & $22(7.7)$ & Bursera fagaroides & $0.18(7)$ \\
Acacia macracantha & $20(7)$ & Apoplanesia paniculata & $2.67(100)$ \\
Total & $285(100)$ & Total & \\
\hline & & &
\end{tabular}

como anota Gentry (1995), puede deberse a sesgos en el muestreo regional. Lo anterior es factible, ya que, por ejemplo, Croton presenta especies con altos valores de importancia en Infiernillo y en El Limón, localidades de la Cuenca del Balsas con BTC censadas por Trejo (1998).

Los sitios estudiados en El Tarimo concuerdan con trabajos previos que señalan a Leguminosae como la familia más rica en especies (Lott et al., 1987; Gentry, 1988, 1995; Ray et al., 1998; Sabogal y Valerio, 1998; Trejo, 1998; Gilliespie et al., 2000; Phillips y Miller, 2002; Salas-Morales, 2002; Sánchez-Velázquez et al., 2002; Gallardo-Cruz et al., 2005; Lott y Atkinson, 2006); hasta el momento, sólo en 3 localidades paleotropicales no se ha observado este patrón: Belinga, Gabón; Ogun, Nigeria, y Nueva Caledonia, donde Euphorbiaceae, Rubiaceae y Sapindaceae, respectivamente, son las familias más sobresalientes (Phillips y Miller, 2002; Gillespie y Jafré, 2003). En segundo lugar está Burseraceae, lo que coincide con los resultados obtenidos en 3 de los 4 sitios muestreados por Trejo (1998) en las inmediaciones de la cuenca del río Balsas y para Beza 2, Madagascar, África (Phillips y Miller, 2002). Las familias que ocupan las siguientes posiciones en jerarquía en El Tarimo (Rubiaceae, Anacardiaceae y Boraginaceae) también se han registrado entre las más diversas para los BTC de América (Gentry, 1995; Salas-Morales, 2002; Trejo, 1998; Gilliespie et al., 2000; Phillips y Miller, 2002).

La familia Leguminosae fue también la predominante 
Cuadro 4. Número de familias y especies, número de individuos ( $\geq 2.5 \mathrm{~cm}$ d.a.p.), área basal $\left(\mathrm{AB}, \mathrm{m}^{2}\right)$ e índice $\alpha$ de Fisher en las 5 localidades con el mayor y el menor número de las especies conocidas en el mundo para el bosque tropical caducifolio (tomado de Phillips y Miller, 2002) y para los 4 sitios incluidos en el presente estudio (en negritas). La información obtenida de este trabajo sólo consideró aquellos sitios con un área de muestreo de 0.1 ha y con datos para todas las especies leñosas. Las localidades se arreglaron con base en su valor de especies.

\begin{tabular}{lccccc}
\hline Localidad & Especies & Familias & Individuos & AB & $\alpha$ \\
\hline Río Negro (Bolivia) & 169 & 52 & 331 & 3.51 & 138.3 \\
Nuevo Mundo (Bolivia) & 149 & 41 & 358 & 4.49 & 95.8 \\
Makokou 1 (Gabón) & 134 & 40 & 322 & 3.67 & 87.5 \\
Ndakan upland (República Centroafricana) & 121 & 39 & 383 & 7.48 & 60.9 \\
Loma de Los Colorados (Colombia) & 120 & 41 & 492 & 3.40 & 50.6 \\
Sitio 3 El Tarimo (México) & $\mathbf{5 0}$ & $\mathbf{2 2}$ & $\mathbf{3 0 0}$ & $\mathbf{3 . 6 4}$ & $\mathbf{1 7 . 1}$ \\
Cerro de Santiago (Bolivia) & 47 & 27 & 179 & 1.43 & 20.8 \\
Pande Forest Reserve (Tanzania) & 46 & 16 & 149 & 1.02 & 22.8 \\
Los Haitaíses National Park (República Dominicana) & 42 & 29 & 176 & 3.45 & 17.5 \\
Sitio 2 El Tarimo (México) & $\mathbf{4 0}$ & $\mathbf{1 9}$ & $\mathbf{2 8 9}$ & $\mathbf{3 . 5 2}$ & $\mathbf{1 2 . 6}$ \\
Sitio 4 El Tarimo (México) & $\mathbf{4 0}$ & $\mathbf{1 9}$ & $\mathbf{1 8 8}$ & $\mathbf{3 . 1 8}$ & $\mathbf{1 5 . 5}$ \\
Estación Biológica de Los Llanos (Venezuela) & 38 & 21 & 160 & 1.19 & 15.8 \\
Sitio 1 El Tarimo (México) & $\mathbf{3 6}$ & $\mathbf{1 7}$ & $\mathbf{2 1 1}$ & $\mathbf{3 . 4 2}$ & $\mathbf{1 2 . 4}$ \\
Salta (Argentina) & 25 & 15 & 197 & 2.37 & 7.6 \\
\hline
\end{tabular}

entre las 3 formas de crecimiento analizadas, lo cual concuerda con los sitios estudiados en la costa de Oaxaca por Salas-Morales (2002) y para la forma de crecimiento arbórea y arbustiva en la región de Chamela-Cuixmala, Jalisco (Lott y Atkinson, 2006). Llama la atención la predominancia de Leguminosae para el caso de las lianas, ya que Bignoniaceae se menciona generalmente como la familia con mayor riqueza de especies para esta forma de crecimiento en el BTC (Lott et al., 1987; Gentry, 1991, 1995; Trejo, 1998; Gilliespie et al., 2000), si bien en estos estudios Leguminosae se ubica siempre entre las primeras posiciones. El número de especies encontrado con un d.a.p. $\geq 1 \mathrm{~cm}$ en El Tarimo es similar cuando se le compara con 2 de las localidades de BTC muestreadas en México por Trejo y Dirzo (2002) (Alamos, Sonora, 46 especies; Cerro Zináparo, Michoacán, 48 especies). Está situación es idéntica cuando se revisa la riqueza de familias. Un contraste más marcado se presenta cuando se comparan las cifras mencionadas para la zona de estudio con los datos registrados para la cuenca del río Balsas, particularmente con los de Infiernillo, donde Trejo y Dirzo (2002) encontraron 115 especies y 38 familias.

La comparación de atributos de la estructura del bosque, específicamente número de individuos y área basal (Cuadro 3), no modifican lo descrito anteriormente con respecto al número de especies y familias, pues los valores que se mencionan para México por Trejo (1998) oscilan entre 443 (El Limón, Morelos, Cuenca del Balsas) a 777 para el número de individuos (Caleta, Michoacán) y entre 1.85 (Calipam, Puebla) a $10.31 \mathrm{~m}^{2}$ para el área basal (Las Flores, Tamaulipas). El contraste entre los sitios estudiados con otras localidades censadas por el método de Gentry a nivel global (Cuadro 4), muestra claramente que la riqueza taxonómica, el número de individuos y el área basal ubican a El Tarimo como una región poco relevante. Los valores del índice de diversidad alfa de Fisher para el valor diamétrico $\geq$ de $1 \mathrm{~cm}$ o el de $2.5 \mathrm{~cm}$, refuerzan la conclusión de baja diversidad. Sin embargo, si se calcula el número de especies en una zona como El Tarimo, utilizando la fórmula propuesta por Gentry (1982), en la que se presenta como variable predictora la precipitación promedio anual, su riqueza esperada sería del orden de 48 especies $\{\mathrm{S}=-1.2+0.05676(872.3)\}$, lo cual concuerda con los valores obtenidos (Cuadro 4).

La información documentada para la flora y vegetación de las especies leñosas de El Tarimo confirman algunos 
de los patrones indicados en México y a nivel global para el BTC; por ejemplo, la importancia de la familia Leguminosae o la predominancia de las especies árboreas y arbustivas respecto a la riqueza de lianas. De igual manera, confirma la abundancia de algunas especies previamente registradas en la literatura como miembros dominantes de este tipo de vegetación en la Cuenca del Balsas, como Amphypterigium adstringens, Cochlospermum vitifolium, Cordia elaeagnoides, Cyrtocarpa procera, Pseudobombax ellipticum y Randia capitata (Miranda, 1947; Rzedowski, 1978; Trejo, 1998) y la importancia florística y biogeográfica del género Bursera (Rzedowski et al., 2005), con 4 especies endémicas de la Cuenca del Balsas registradas en los sitios de El Tarimo (B. coyucensis, $B$. crenata, B. discolor y B. velutina).

El propósito final del presente estudio es despertar el interés por realizar estudios similares para otras localidades de la región del río Balsas o de otras regiones con BTC de México, los cuales pueden ser piezas importantes para la definición de políticas de manejo más adecuadas de los recursos vegetales que alberga. Ante la fuerte presión que las actividades humanas están provocando en las comunidades con BTC en esta provincia florística, es deseable que estos estudios se realicen en el mínimo plazo posible.

\section{Agradecimientos}

Los autores del trabajo estamos sumamente agradecidos con Juan Martínez Cruz, quien colaboró de manera entusiasta con el trabajo de campo y con el Dr. Jerzy Rzedowski, por su apoyo en la determinación de especies de Burseraceae.

\section{Literatura citada}

Balvanera, P., E. Lott, G. Segura, C. Siebe y A. Islas. 2002. Patterns of ß-diversity in a Mexican tropical dry forest. Journal of Vegetation Sciencie 13: 145-158.

Ceballos, G. y A. García. 1995. Conserving Neotropical biodiversity: the role of dry forest in western México. Conservation Biology 9: 1349-1353.

Challenger, A. 1998. Utilización y conservación de los ecosistemas terrestres de México: pasado, presente y futuro. Comisión Nacional para Conocimiento y Uso de la Biodiversidad, Instituto de Biología, UNAM y Agrupación Sierra Madre, México, D. F. 847 p.

Cuevas G., R., N. Núñez L., L. Guzmán H. y F. Santana M. 1998. El bosque tropical caducifolio en la Reserva de la Biosfera Sierra de Manantlán, Jalisco-Colima,
México. Boletín Instituto de Botánica de la Universidad de Guadalajara 1-3: 445-491.

Ferrusquía-Villafranca, I. 1998. Geología de México: una sinopsis. In Diversidad biológica de México: orígenes y distribución, T. P. Ramamoorthy, R. Bye, A. Lot y J. Fa (eds.) Instituto de Biología, UNAM, México, D. F. p. 3-108.

Gallardo-Cruz, J. A., J. A. Meave y E. A. Pérez-García. 2005. Estructura, composición y diversidad de la selva baja caducifolia del cerro Verde, Nizanda (Oaxaca), México. Boletín de la Sociedad Botánica de México 76: 19-35.

García, E. 1981. Modificaciones al sistema de clasificación de Köppen. Tercera edición. Larios, México, D. F. $252 \mathrm{p}$.

Gentry, A. H. 1982. Patterns of neotropical plant species diversity. Evolutionary Biology 15: 1-84.

Gentry, A. H. 1988. Changes in plant community diversity and floristic composition on environmental and geographical gradients. Annals of the Missouri Botanical Garden 75: 1-34.

Gentry, A. H. 1991. The distribution and evolution of climbing plants. In The biology of vines, F. Putz y $\mathrm{H}$. A. Mooney (eds.). Cambridge University Press. p. 3-49.

Gentry, A. H. 1995. Diversity and floristic composition of neotropical dry forests. In Seasonally dry tropical forests, S. H. Bullock, H. A. Mooney y E. Medina (eds.) Cambridge University Press, Cambridge. p. 146-190.

Gentry, H. S. 1942. Rio Mayo Plants. A study of the flora and vegetation of the Valley of the Rio Mayo, Sonora. Carnegie Institution of Washington Publication 527. Washington, D.C. 328 p.

Gilliespie, T. W., A. Grijalva y N. Farris. 2000. Diversity, composition, and structure of tropical dry forests in Central America. Plant Ecology 147: 37-47.

Gilliespie, T. W. y T. Jafré. 2003. Tropical dry forest in New Caledonia. Biodiversity and Conservation 12: 1687-1697.

INEGI (Instituto Nacional de Estadística Geografía e Informática). 1989. Guías para la interpretación de cartografía. Edafología. México. 45 p.

INEGI (Instituto Nacional de Estadística, Geografía e Informática). 2001. Anuario estadístico del estado de Guerrero. Instituto Nacional de Estadística, Geografía e Informática. México. 592 p.

Lott, E., S. H. Bullock y J. A. Solis-Magallanes. 1987. Floristic diversity and structure of upland and Arroyo Forests of coastal Jalisco. Biotropica 19: 228-235.

Lott, E. J. y T. H. Atkinson. 2006. Mexican and Central American seasonally dry tropical forests: ChamelaCuixmala, Jalisco, as a focal point for comparison. 
In Neotropical savannas and seasonally dry forests. Plant diversity, biogeography, and conservation, R. T. Pennington, G. P. Lewis, and J. A. Ratter (eds.). Taylor \& Francis, Florida. p. 315-342.

Magurran, A. E. 2004. Measuring biological diversity. Blackwell, Oxford. 256 p.

Martínez-Yrízar, A., A. Búrquez y M. Maass. 2000. Structure and functioning of tropical deciduous forest in western Mexico. In The tropical deciduous forest of Alamos. Biodiversity of a threatened ecosystem in Mexico, R. H. Robichaux y D. A. Yetman (eds.). The University of Arizona, Tucson. p. 19-35.

Masera, O., M. J. Ordóñez y R. Dirzo. 1997. Carbon emissions from Mexican forests: current situation and long term scenarios. Climatic Change 35: 256-259.

Miranda, F. 1941. Estudios sobre la vegetación de México I. La vegetación de los cerros al sur de la meseta de Anáhuac-El Cuajiotal. Anales del Instituto de Biología, Universidad Nacional Autónoma de México 12: 569614.

Miranda, F. 1947. Estudios sobre la vegetación de México. V. Rasgos de la vegetación de la Cuenca del Río Balsas. Revista de la Sociedad Mexicana de Historia Natural 1-4: 95-114.

Miranda, F. y E. Hernández-X. 1963. Los tipos de vegetación de México y su clasificación. Boletín de la Sociedad Botánica de México 28: 29-178.

Mueller-Dumbois, D. y H. Ellenberg. 1974. Aims and methods of vegetation ecology. John Wiley, New York. 545 p.

Murphy, P.G. y A. E. Lugo. 1986. Ecology of tropical dry forest. Annals Review of Ecology and Systematics 17: 67-88.

Palacio-Prieto, J. L., G. Bocco, A. Velásquez, J. F. Mas, F. Takaki-Takaki, A. Victoria, L. Luna-González, G. Gómez-Rodríguez, J. López-García, M. Palma M., I. Trejo-Vázquez, A. Peralta H., J. Prado-Molina, A. Rodríguez-Aguilar, R. Mayorga-Saucedo y F. González M. 2000. La condición actual de los recursos forestales en México: Resultados del Inventario Forestal Nacional 2000. Boletín del Instituto de Geografía 43: 183-203.

Pennington, T. D. y J. Sarukhán. 1998. Árboles tropicales de México. Manual para la identificación de las principales especies. Segunda edición. Universidad Nacional Autónoma de México y Fondo de Cultura Económica. México, D.F. 521 p.

Phillips, O. y J. S. Miller. 2002. Global patterns of plant diversity: Alwyn H. Gentry's forest transect data set. Monographs in Systematic Botany from the Missouri Botanical Garden 89: 1-319.

Ray, G. J., F. Dallmeier, y J. A. Comiskey. 1998. The structure of two subtropical dry forest communities on the island of St John, US Virgin Islands. In Forest biodiversity in North, Central and South America, and the Caribbean, Man and the Biosphere series 21, F. Dallmeier and J. A. Comiskey (eds.). UNESCO, Paris. p. 367-384.

Rzedowski, J. 1978. La vegetación de México. Limusa, México, D. F. 432 p.

Rzedowski, J. 1990. Vegetación potencial. Atlas Nacional de México. Instituto de Geografía, UNAM, México, D. F.

Rzedowski, J. 1991a. Diversidad y orígenes de la flora fanerogámica de México. Acta Botanica Mexicana 14: 3-21.

Rzedowski, J. 1991b. El endemismo en la flora fanerogámica mexicana: una apreciación analítica preliminar. Acta Botanica Mexicana 15: 47-64.

Rzedowski, J., R. Medina L. y G. Calderón de Rzedowski. 2005. Inventario del conocimiento taxonómico, así como de la diversidad y del endemismo regionales de las especies mexicanas de Bursera (Burseraceae). Acta Botanica Mexicana 70: 85-111.

Sabogal, C. y L. Valerio. 1998. Forest composition, structure, and the regeneration in a dry forest of the Nicaraguan Pacific Coast. In Forest biodiversity in North, Central and South America, and the Caribbean, Man and the Biosphere series 21, F. Dallmeier y J. A. Comiskey (eds.). UNESCO, Paris. p. 187-212.

Salas-Morales, S. H. 2002. Relaciones entre la heterogeneidad ambiental y la variabilidad estructural de las selvas tropicales secas de la costa de Oaxaca, México. Tesis, Maestría (Biología Ambiental) Facultad de Ciencias, Universidad Nacional Autónoma de México, México, D. F. 101 p.

Sánchez-Velásquez, L. R., G. Hernández-Vargas, M. A. Carranza-M, M. R. Pineda-López, R. Cuevas G. y F. Aragón-C. 2002. Estructura arbórea del bosque tropical caducifolio usado para la ganadería extensiva en el norte de la Sierra de Manantlán, México. Antagonismo de usos. Polibotánica 13: 25-46.

SPP (Secretaría de Programación y Presupuesto). 1983. Carta Edafológica E14-4, escala 1:250,000. México.

Trejo, I. 1998. Distribución y diversidad de selvas bajas de México: relaciones con el clima y el suelo. Tesis doctoral. División de Estudios de Posgrado. Facultad de Ciencias, Universidad Nacional Autónoma de México, México. 206 p.

Trejo, I. y R. Dirzo. 2000. Deforestation of seasonally dry tropical forest: a national and local analysis in Mexico. Biological Conservation 94: 133-142.

Trejo, I. y R. Dirzo. 2002. Floristic diversity of Mexican seasonally dry tropical forest. Biodiversity and 
Conservation 11: 2063-2084.

White, D. A. y C. S. Hood. 2004. Vegetation patterns and environmental gradients in tropical dry forests of the northern Yucatan Peninsula. Journal of Vegetation Science 15: 151-160.

Zar, J. H. 1996. Biostatistical analysis. Prentice Hall, New
Jersey. $121 \mathrm{p}$.

Zepeda, G. C. y E. Velázquez M. 1999. El bosque tropical caducifolio de la vertiente sur de la sierra de Nanchititla, estado de México: la composición y la afinidad geográfica de su flora. Acta Botánica Mexicana 46: 29-55.

Apéndice l. Lista de familias y especies $\geq 1 \mathrm{~cm}$ d.a.p. censadas en el bosque tropical caducifolio El Tarimo, indicando su forma de crecimiento y presencia $(*)$ por sitio.

\begin{tabular}{llll}
\hline Familia y especies & $\begin{array}{l}\text { Forma de } \\
\text { crecimiento }\end{array}$
\end{tabular}$\quad$ Sitio $1 \quad$ Sitio $2 \quad$ Sitio $3 \quad$ Sitio 4

\section{ANACARDIACEAE \\ Comocladia engleriana Loes. \\ Cyrtocarpa procera Kunth \\ Pseudosmodingium perniciosum (Kunth) Engl. \\ Spondias purpurea L.}

\section{APOCYNACEAE}

Plumeria rubra L.

Thevetia peruviana (Pers.) K. Schum.

\section{ASCLEPIADACEAE}

Marsdenia lanata (Paul G. Wilson) W.D. Stevens

M. macrophylla (Humb. et Bonpl. Ex Schult.) E. Fourn.

BIGNONIACEAE

\section{Crescentia alata Kunth}

Tabebuia impetiginosa (Mart. ex DC.) Standl.

\section{BIXACEAE}

Cochlospermum vitifolium (Willd.) Spreng.

\section{BOMBACACEAE}

Ceiba aesculifolia (Kunth) Britten et Barker f.

Pseudobombax ellipticum (Kunth) Dugand

\section{BORAGINACEAE}

Cordia alliodora (Ruiz et Pav.) Oken

C. dentata Poir.

C. dodecandra DC.

C. elaeagnoides DC.

\section{BURSERACEAE}

Bursera coyucensis Bullock

B. crenata P.G. Wilson

$B$. discolor Rzed.

B. fagaroides (Kunth) Engl.

B. grandifolia (Schltdl.) Engl.

$B$. heteresthes Bullock

B. sarukhanii Guevara et Rzed.

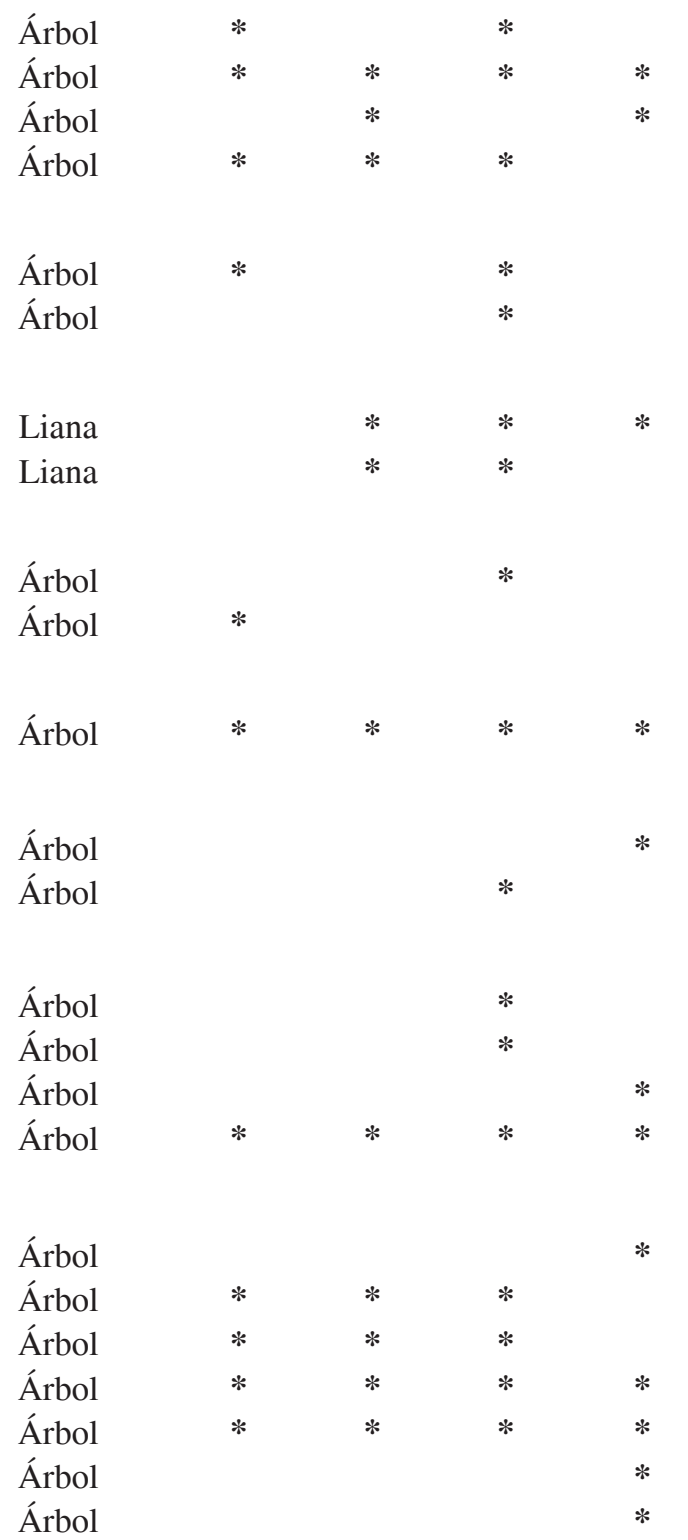


B. submoniliformis Engl.

Árbol

B. velutina Bullock

Árbol

CACTACEAE

Pachycereus pecten-aboriginum (Engelm. ex S. Watson)

Britton et Rose

Stenocereus sp.

Árbol

Árbol

COMPOSITAE

Otopappus mexicanus (Rzed.) H. Rob.

Trixis mexicana Lex.

Liana

Liana

CONVOLVULACEAE

Ipomoea sp. 1

Liana

Ipomoea sp. 2

Liana

\section{ERYTHROXYLACEAE}

Erythroxylum mexicanum Kunth

E. rotundifoluim Lunan

\begin{tabular}{|c|c|c|c|}
\hline Arbusto & $*$ & $*$ & $*$ \\
\hline Arbusto & $*$ & $*$ & $*$ \\
\hline Arbusto & $*$ & $*$ & * \\
\hline Arbusto & & & \\
\hline Liana & & & $*$ \\
\hline Árbol & $*$ & $*$ & $*$ \\
\hline Árbol & $*$ & $*$ & $*$ \\
\hline Árbol & $*$ & & \\
\hline Árbol & $*$ & $*$ & $*$ \\
\hline Arbusto & $*$ & & \\
\hline Arbusto & & & \\
\hline Árbusto & & $*$ & \\
\hline Arbusto & & $*$ & \\
\hline Liana & & & $*$ \\
\hline Árbol & $*$ & & $*$ \\
\hline Arbusto & $*$ & & $*$ \\
\hline Arbusto & & & $*$ \\
\hline Liana & & & $*$ \\
\hline Árbol & $*$ & $*$ & $*$ \\
\hline Árbol & $*$ & $*$ & $*$ \\
\hline Árbol & $*$ & $*$ & $*$ \\
\hline Árbol & & $*$ & $*$ \\
\hline Liana & $*$ & & $*$ \\
\hline Arbusto & $*$ & $*$ & $*$ \\
\hline \multicolumn{4}{|l|}{ Arbusto } \\
\hline Liana & $*$ & & $*$ \\
\hline Arbusto & & $*$ & \\
\hline Árbol & $*$ & & $*$ \\
\hline
\end{tabular}

\section{EUPHORBIACEAE}

Euphorbiaceae 1

Euphorbiaceae 2

HIPPOCRATACEAE

Hippocratea celastroides Kunth

JULIANACEAE

Amphipterygium adstringens (Schltdl.) Standl.

\section{LEGUMINOSAE}

Acacia macracantha Humb. et Bonpl. ex Willd.

Apoplanesia paniculata C. Presl.

Bauhinia ungulata $\mathrm{L}$.

Caesalpinia coriaria (Jacq.) Willd.

C. pulcherrima (L.) Sw.

Chamaecrista absus (L.) H.S. Irwin et Barneby

Desmodium sp.

Entada polystachya (L.) DC.

Haematoxylum brasiletto H. Karst.

Leguminosae 1

Leguminosae 2

Leguminosae 3

Lonchocarpus eriocarinalis Micheli

Lysiloma microphyllum Benth.

L. tergeminum Benth.

Mimosa sp.

Nissolia sp.

Phitecellobium unguis-cati (L.) Benth.

Phitecellobium sp.

Piptadenia flava (Spreng. ex DC.) Benth.

Platymiscium lasiocarpum Sandw.

Poeppigia procera C. Presl

Árbol 
Piscidia piscipula (L.) Sarg.

Árbol

Rynchosia sp.

Senna pallida (Vahl) H.S. Irwin et Barneby

Liana

Arbusto

MALPIGHIACEAE

Bunchosia palmeri S. Watson

Gaudichaudia albida Schltdl. et Cham.

Arbusto

MORACEAE

Ficus cotinifolia Kunth

Liana

Árbol

RHAMNACEAE

Karwinskia humboldtiana (Willd. ex Roem. et Schult.) Zucc.

Arbusto

\section{RUBIACEAE}

Exostema mexicanum A. Gray

Genipa americana L.

Guettarda elliptica Sw.

Psychotria horizontalis Sw.

Randia capitata DC.

R. echinocarpa Moc. et Sessé ex DC.

Árbol

Árbol

Arbusto

Arbusto

Arbusto

Arbusto

\section{SAPINDACEAE}

Cardiospermum halicacabum L.

Serjania triquetra Radlk.

$\begin{array}{lllll}\text { Liana } & * & & * & * \\ \text { Liana } & * & * & * & * \\ \text { Árbol } & * & * & & \\ & & & & \\ \text { Árbol } & & * & & * \\ \text { Árbol } & & * & * & \\ & & & & \\ \text { Liana } & * & * & * & * \\ \text { Liana } & * & * & * & *\end{array}$

TILIACEAE

Heliocarpus pallidus Rose

VERBENACEAE

Vitex mollis Kunth.

Vitex pyramidata B. L. Rob.

Liana

Ampelocissus acapulcensis (Kunth) Planch.

Cissus sicyoides L.

Arbusto

Indeterminada sp. 1

Arbusto

Indeterminada sp. 2 